\title{
Looking for Changes in the Heart Rate of Patients with Neurocardiogenic Syncope
}

\author{
Fatima MHSP Silva ${ }^{1}$, Mariana A Leite 2 , Julio C Crescencio², Antonio Carlos Silva Filho ${ }^{3}$, \\ Lourenço Gallo Junior ${ }^{2}$ \\ ${ }^{1}$ Pharmaceutical Sciences School of Ribeirao Preto, University of Sao Paulo, Ribeirao Preto, Brazil \\ ${ }^{2}$ Medical School of Ribeirao Preto, University of Sao Paulo, Ribeirao Preto, Brazil \\ ${ }^{3}$ Centro Universitario de Franca, Uni-FACEF, Franca, Brazil
}

\begin{abstract}
Neurocardiogenic Syncope (NCS) is a temporary loss of consciousness due to a global cerebral hypoperfusion caused by bradycardia and/or significant hypotension. We analyzed the heart rate variability (HRV) of three groups during the Tilt-test, in the supine and standing position, before hypotension. The groups are: 1) 17 healthy individuals, 2) 18 patients with complaint of NCS, but a negative Tilt-test and 3) 16 patients with complaint of NCS and a positive Tilt-test. There is no significant difference for age and anthropometric characteristics among groups (5\%). We did multifractal analysis by the MFDFA technique. We estimated, also, the Largest Lyapunov Exponent (LLE), using the Rosenstein algorithm. The loss of multifractality, as well as a decrease in the LLE, imply less complex system. The multifractal analysis showed no difference among groups, in both the supine and in the standing position (5\%). However, compared to LLE, there are differences among groups, in the standing position, but not in the supine. There is significant difference between the supine and standing position for all groups. Most notably, the LLE for groups 2 and 3 are lower than for the group 1. In addition, groups 2 and 3 are equal. We conclude that in the standing position, there is a reduction of complexity of the HRV for patients with NCS (5\%).
\end{abstract}

\section{Introduction}

The NCS is a syndrome characterized by transient loss of consciousness due to a global cerebral hypoperfusion; it is quick start, short duration and spontaneous recovery. It is a common clinical condition, with an incidence up to $35 \%$ of the population. It is benign, however, in $30 \%$ of patients with NCS, the episodes are recurrent and decrease their quality of life by restricting the daily activities and professional life [1-2].

Are there significant changes in the HRV of patients with NCS symptom?
In this work, we propose to investigate whether multifractal analysis, or another nonlinear parameter of dynamical systems theory, the LLE, are useful mathematical tools to detect changes in the dynamical of the heart rate of patients with NCS in order to support the medical diagnosis. This is another exploration of nonlinear dynamics parameters by our research group [3-9] always with a noninvasive goal in mind.

The time between successive $\mathrm{R}$ peaks obtained from ECG is called RR interval. The sequence of RR intervals forms a time series called RR series, which reveals characteristics that to certain scales are approximately similar to those of self-similarity processes. Such processes are fractals.

It is possible that the different levels of fractality are associated with different states of health. Systems that are more complex have degree of multifractality greater than the most organized. Multifractal analysis, for example, has been used successfully to discriminate healthy individuals from patients. As the heart rate has greater complexity in healthy individuals, the loss of multifractality points to a disease state [8, 10-14].

Another important parameter of nonlinear dynamics is the Lyapunov exponent. This measures the divergence rate (or convergence) of trajectories initially next in a phase space and estimates the amount of system sensitivity to initial conditions. In addition, a decrease of the LLE is considered, also, as an attempt of the system in organize itself, which can occur in the presence of some disease [7, 15-17].

Many researchers have developed algorithms to estimate the LLE. The Rosenstein algorithm is accurate because it takes the advantage of all the available data. We calculated the LLE in each RR series based on the Roseinstein algorithm [18-21], which allows the calculation, on small data sets, which is our case (on average, 1211 points in the supine position and 1432 points in standing position).

\section{Methods}




\subsection{Subjects and data acquisition}

We studied 51 individuals in these three groups: 1) 17 healthy subjects (control group), 12 women and 5 men; 2) 18 patients with negative Tilt-test for NCS, 11 women and 7 men; and 3) 16 patients with positive Tilt-test for NCS, 15 women and 1 men. We did not observe a statistically significant difference in the comparison of age and anthropometric characteristics among individuals in groups 1, 2 and 3.

Each one was subjected to the Tilt-test protocol that consisted in these three phases:

1. Pre-Tilt: the patient lays on a Tilt-table with footrest for 20 minutes in the supine horizontal position; 2 . Tilt: the table has tilted to 70 degrees vertically in less than 10 seconds, with a maximum duration of 45 minutes; it is immediately discontinued if the patient presents any sign of syncope. In this case, the table quickly returns to the horizontal or Trendelenburg position [22]; 3. Post-Tilt: the patient returns to the supine horizontal position. The phases that were used for data collection were the pre-Tilt (supine) and Tilt (standing) - the length of the excerpts varies from 1,000 to 1,500 points, prior to syncope.

The electrical signal of the electrocardiogram (ECG) was filtered (high-pass: $100 \mathrm{~Hz}$, low-pass: $0.5 \mathrm{~Hz}$ ) and amplified by a universal bioelectric amplifier (7754A, Hewlett Packard, Palo Alto, California, USA). Then, the ECG signals are processed by an analog/digital converter (DI- 720, DATAQ Instruments, Akron, Ohio, USA) and sampled (1000 Hz per channel) through Windaq PRO + software (DATAQ Instruments, Akron, Ohio, USA). Files with ECG records had processed by the Windaq software, able to produce files in text format (ASCII). This process allowed the generation of time series with RR interval values used in our study.

\subsection{Multifractal analysis}

The MFDFA method [8, 10-14] was applied in Matlab code in order to estimate the singularity spectrum $\mathrm{D}\left(\mathrm{h}_{\mathrm{q}}\right)$ to each RR series. The width of the spectrum given by the difference between the maximum and minimum $h_{q}\left(\Delta h_{q}\right)$ measures the degree of multifractality in the series. The input parameters were: signal $=\mathrm{RR}$ series; scale $=[6,8$, $10, \ldots, 64]$; q (q-order that weights the local variations) $=$ $[-5,-4,-3,-2,-1,0,1,2,3,4,5]$; m (polynomial order for the detrending) $=1$.

\subsection{LLE analysis}

The LLE was calculated in C code written by Rainer Hegger, based on Roseinstein's algorithm. This C code mentioned was extracted from TISEAN 3.0.1 (Time Series Analysis), free software [20]. The input parameters were RR series, its corresponding delay $(\tau)$ of the Takens [21], estimated by the autocorrelation function, embedding dimension $\mathrm{m}=10$; window around the reference point which should be omitted $t=100$, minimal length scale for the neighborhood search $r=0.01$ and the number of iterations $s=15$. Finally, the LLE was estimated by linear regression of the logarithm of the stretching factor [18].

\subsection{Statistical analysis}

Once satisfied the conditions of normality, homogeneity of variances and independence between samples, we carry out the one-way ANOVA to compare the $\Delta \mathrm{h}_{\mathrm{q}}$ and LLE among the three groups of subjects in the same position.

The one-sided t-test for paired samples was used to compare the values of $\Delta \mathrm{h}_{\mathrm{q}}$ and LLE in the supine position with those in the standing position in each group.

\section{Results}

\subsection{Results of multifractal analysis}

We observed the singularity spectrum, $\mathrm{D}\left(\mathrm{h}_{\mathrm{q}}\right)$, as well as the degree of multifractality given by the width of the spectrum $\left(\Delta h_{q}\right)$ for each RR series. The average $\Delta h_{q}$ in the groups are showed in Table 1 with their respective standard deviations.

Table 1 . The $\Delta \mathrm{h}_{\mathrm{q}}$ in the groups.

\begin{tabular}{clclc}
\hline & \multicolumn{2}{c}{ Supine } & \multicolumn{2}{c}{ Standing } \\
\cline { 2 - 5 } Group & $\overline{\Delta \mathbf{h q}}$ & SD & $\Delta \mathbf{h q}$ & SD \\
\hline 1 & 0.312 & 0.237 & 0.556 & 0.214 \\
\hline 2 & 0.395 & 0.198 & 0.491 & 0.210 \\
\hline 3 & 0.262 & 0.148 & 0.441 & 0.280 \\
\hline
\end{tabular}

We were able to prove statistically, with degree of significance of $5 \%$, that $\Delta \mathrm{h}_{\mathrm{q}}$ is higher in the standing position (Table 2).

Table 2. Comparison of $\Delta \mathrm{h}_{\mathrm{q}}$ in the supine position with the standing position.

\begin{tabular}{ccc}
\hline Group & $\mathrm{n}$ & $\mathrm{p}$-value \\
\hline 1 & 17 & 0.0052 \\
2 & 18 & 0.0416 \\
3 & 16 & 0.0054 \\
\hline
\end{tabular}

The Figure 1 shows the spectrum $\mathrm{D}\left(\mathrm{h}_{\mathrm{q}}\right)$ in the supine position compared with the spectrum in the standing position of the same individual. Notice that the parable drawn on the lower chart (standing position) is more open 
than the parable on the upper chart, featuring a large increase in multifractality [14]. We could observe that for all individuals.

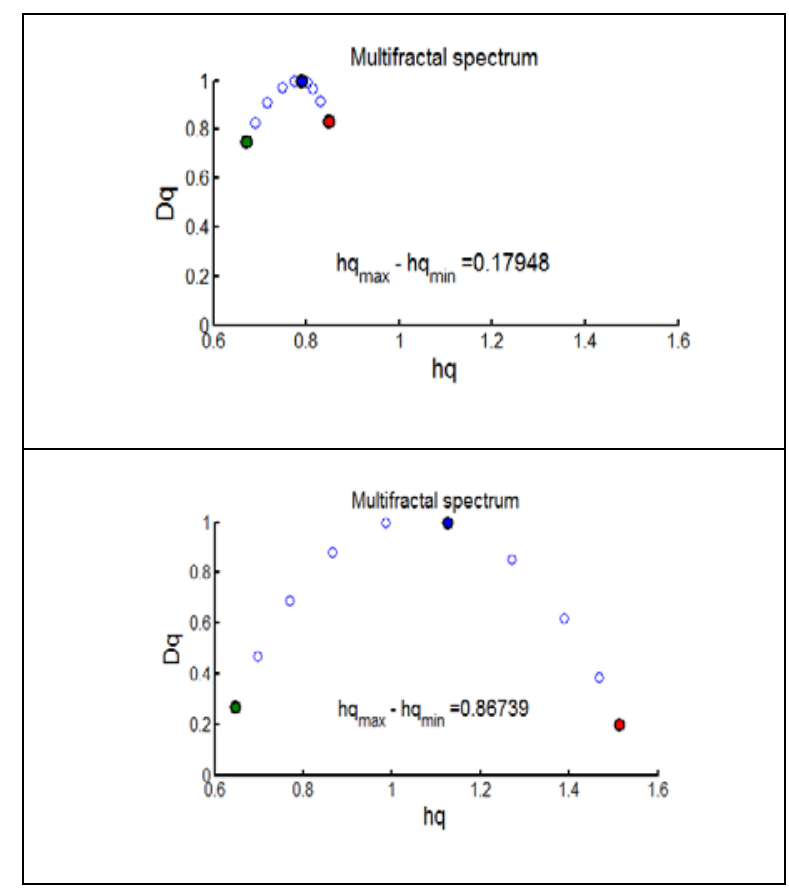

Figure 1. Multifractal Spectrum of an individual from the group 1 in the supine position (upper graph) and in the standing position (lower graph).

However, by the multifractal analysis, we could not find statistically significant differences among groups by ANOVA, at the level of 5\% in the supine position (p-value $=0.151)$ nor in the standing position ( $\mathrm{p}$-value $=0.622)$.

\subsection{Results of LLE analysis}

The Table 3 shows the average LLE in the groups with their respective standard deviations.

Table 3. The LLE in the groups.

\begin{tabular}{ccccc}
\hline Group & \multicolumn{2}{c}{ Supine } & \multicolumn{2}{c}{ Standing } \\
& LLE & SD & $\overline{\text { LLE }}$ & SD \\
\hline 1 & 0.0947 & 0.0094 & 0.1210 & 0.0160 \\
\hline 2 & 0.0917 & 0.0072 & 0.1064 & 0.0149 \\
\hline 3 & 0.0906 & 0.0078 & 0.1104 & 0.0154 \\
\hline
\end{tabular}

We were able to prove statistically that LLE are higher in the standing position than in the supine (p-value $<0.01$, in all comparisons).
In addition, we might find statistically significant differences among groups in the standing position (ANOVA, $\mathrm{p}$-value $=0.022<0.05$ )

Then, we proceed with multiple comparisons in the standing position (Table 4).

Table 4. Multiple comparisons in the standing position

\begin{tabular}{cc}
\hline Comparisons & p-value \\
\hline $1-2$ & 0.004 \\
$1-3$ & 0.031 \\
$2-3$ & 0.229 \\
\hline
\end{tabular}

Looking at Table 4, we can observe significant difference between groups 1 and 2 and between groups 1 and $3(5 \%)$, but is not significant difference between groups 2 and 3.

However, we could not find statistically significant differences among groups in the supine position by ANOVA, at the level of 5\% (ANOVA, p-value $=0.151)$.

\section{Conclusions}

Looking at the multifractal spectrum of individuals in the supine position and comparing them with the spectrum in the standing position, we conclude that on average the degrees of multifractality are higher in the standing position, which implies higher complexity of the system. This increase in system complexity in this position was also confirmed by the significant increase of LLE.

However, we found no significant differences in the HRV between the groups of individuals by multifractal analysis.

Moreover, in standing position, we can say that the LLE is significantly higher for Group 1 (control group) than that of group 2 or group 3 . Still talking about the LLE, groups 2 and 3 are statistically equal.

Now, when compared the groups by LLE in the supine position, we did not observe any significant difference (5\%) among them. This may be due to the small number of points.

Finally, we concluded that there is a tendency of the heart rhythm organize itself, which characterizes a dynamic disease, when it comes to patients of the groups 2 and 3. This means that we found changes in heart rate of patients with NCS.

In short, we can investigate if there is signal of the disease in patients with NCS complaint, by using the LLE, which - it is worth pointing out - is a non-invasive procedure.

\section{References}

[1] Moya A et al. Guidelines for the diagnosis and management 
of syncope. Eur Heart J. 2009; Nov; 30(21):2631-71.

[2] Sheldon RS et al. Heart rhythm society expert consensus statement on the diagnosis and treatment of postural tachycardia syndrome, inappropriate sinus tachycardia, and vasovagal syncope. Heart Rhythm 2015; Jun; 12(6):41-63.

[3] Silva FMHSP et al. Identification of Anaerobic Threshold during Dynamic Exercise in Healthy Man using Kolmogorov-Sinai Entropy. Computers in Cardiology 2005; 32:731-4.

[4] Silva Filho AC et al. Using the Lag of Autocorrelation Function in Order to Identify the Anaerobic Threshold During Dynamic Physical Exercise. Computers in Cardiology 2006; 33: 625-8.

[5] Silva FMHSP et al. Modifications in the Heart Dynamics of Patients with Cardiac Disease. Computers in Cardiology 2009; 36:421-4.

[6] Silva Filho AC et al. A New Parameter in the Nonlinear Dynamics of the Heart: The Higher Reconstruction Step. Computers in Cardiology 2010; 37:959-962.

[7] Silva FMHSP et al. Applying Lyapunov Exponents in Heart Rate Time Series to Identify the Anaerobic Threshold in Healthy Men. Computing in Cardiology 2012; 39:629-632.

[8] Silva FMHSP et al. The Loss of Multifractality as Evidence of Impaired Left Ventricular Ejection Fraction in Patients after Acute Myocardial Infarction. Computing in Cardiology 2014; 41:413-6.

[9] Silva Filho AC et al. Trend Strips: a New Tool to Analyze RR Time Series Computers in Cardiology 2014; 41:365-8.

[10] Ivanov PCh, Amaral LAN, Goldberger AL, Havlin S, Rosenblum MG, Struzik ZR, Stanley HE. Multifractality in human heartbeat dynamics. Nature 1999; 399: 461-5.

[11] Gieraltowski J et al. Multifractal Property Assessment in the Very Low Frequency Range, in Subjects with Different Progression of Aortic Valve Stenosis Disease. Computing in Cardiology 2012; 39:921-4.

[12] Kokosińska et al. Fractal Pattern of Heart Rate Variability Revealing Unknown Very Low Frequency Properties. Computing in Cardiology 2015; 42:597-600.

[13] Kantelhard JW, Zschiegner SA, Koscielny-Bunde E, Havlin S, Bunde A, Stanley HE. Multifractal detrended fluctuation analysis of nonstationary time series. Physica A 2002; 316:87-114.
[14] Ihlen EA. Introduction to Multifractal Detrended Fluctuation Analysis in Matlab. Frontiers in Physiology 2012; 3:1-18.

[15] Wolf A et al. Determining Lyapunov exponents from a time series. Physica D 1985; 16:285-317.

[16] Eckmann JP et al. Liapunov exponents from a time series, Physical Review A 1986; 34:4971-4979.

[17] Sato S et al. Practical methods of measuring the generalized dimension and the largest Lyapunov in high dimensional chaotic systems. Progress of Theoretical Physics 1987; 77: 1-5.

[18] Kantz H. A robust method to estimate the maximal Lyapunov exponent of a time series. Physics Letters A 1994; 185: 77-87.

[19] Roseinstein MT, Collins JJ and DeLuca CJ. A practical method for calculating largest Lyapunov exponents from small data sets. Physica D 1993; 65:117-134.

[20] The TISEAN software package at http:www.mpipks dresden.mpg.de/ tisean.

[21] Takens F. Detecting strange attractors in turbulence. In: Rand DA; Young LS, Ed. Dynamical systems and turbulence. Lecture Notes in Mathematics. Berlin: SpringerVerlag, 1981; 898:336-381.

[22] Fitzpatrick AP, Theodorakis G, Vardas P and Sutton R. Methodology of head-up tilt testing in patients with unexplained syncope. J Am Coll Cardiol. 1991; 17(1):125130.

Address for correspondence:

Fatima Maria Helena Simões Pereira da Silva

Department of Physics and Chemistry, Pharmaceutical Sciences School of Ribeirao Preto, University of Sao Paulo.

Av. do Café, s/n, Ribeirão Preto, SP, Brasil.

CEP 14040903.

fatsilva@usp.br 